\title{
Measuring the Attenuation Length of Water in the CHIPS-M Water Cherenkov Detector
}

\author{
F.Amat ${ }^{1}$, P.Bizouard ${ }^{1}$, J.Bryant ${ }^{2}$, T.J.Carroll ${ }^{5}$, S. De Rijck ${ }^{5}$, S.Germani ${ }^{2}$, \\ T.Joyce $^{3}$, B.Kriesten ${ }^{4}$, M.Marshak ${ }^{3}$, J.Meier ${ }^{3}$, J.K.Nelson ${ }^{4}$, A.J.Perch ${ }^{2}$, \\ M.M.Pfützner ${ }^{2}$, R.Salazar ${ }^{5}$, J.Thomas ${ }^{2}{ }^{6}$, J.Trokan-Tenorio $^{6}$, P.Vahle ${ }^{4}$, \\ R.Wade ${ }^{7}$, C.Wendt ${ }^{6}$, L.H.Whitehead ${ }^{2}$, M.Whitney ${ }^{3}$ \\ ${ }^{1}$ Aix Marseille University Saint-Jerome, 13013 Marseille, France \\ ${ }^{2}$ Dept. of Physics and Astronomy, UCL, Gower St, London WC1E 6BT,UK \\ ${ }^{3}$ School of Physics and Astronomy, University of Minnesota, Minneapolis, MN 55455, \\ $U S A$ \\ ${ }^{4}$ Department of Physics, College of William 83 Mary, Williamsburg, Virginia 23187, USA \\ ${ }^{5}$ Department of Physics, University of Texas at Austin, Austin, Texas 78712, USA \\ ${ }^{6}$ Department of Physics, University of Wisconsin, Madison, WI 53706, USA \\ ${ }^{7}$ Avenir Consulting, Abingdon, Oxfordshire, UK
}

\begin{abstract}
The water at the proposed site of the CHIPS water Cherenkov detector has been studied to measure its attenuation length for Cherenkov light as a function of filtering time. A scaled model of the CHIPS detector filled with water from the Wentworth $2 \mathrm{~W}$ pit, proposed site of the CHIPS deployment, in conjunction with a $3.2 \mathrm{~m}$ vertical column filled with this water, was used to study the transmission of $405 \mathrm{~nm}$ laser light. Results consistent with attenuation lengths of up to $100 \mathrm{~m}$ were observed for this wavelength with filtration and UV sterilization alone.
\end{abstract}

Keywords: Water, Attenuation, Neutrino, Cherenkov, Detector, Filtration, Absorption

\section{Introduction}

The CHIPS (CHerenkov Detectors In PitS) R\&D project will investigate

the proof of principle for a novel and very inexpensive water Cherenkov

Preprint submitted to Nuclear Instruments and Methods in Physics November 18, 2016

(C) 2016. This manuscript version is made available under the Elsevier user license http://www.elsevier.com/open-access/userlicense/1.0/ 
detector [1]. Demonstration of a cost in the region of $\$ 200-\$ 300 \mathrm{k}$ per kiloton (kt) is one of the goals of this program of research in order to realize mega-ton scale detectors in the future within realistic financial constraints. A prototype detector of between 3-5 kt of water is proposed for construction 7 milli-radians (mrad) off-axis in the NuMI neutrino beam, in a flooded mine pit $708 \mathrm{~km}$ from the neutrino source at Fermilab. The prototype detector will observe neutrinos via the detection of Cherenkov radiation produced by their interactions in the water inside the detector.

A major part of the cost saving in the CHIPS concept is achieved by enclosing the detector water volume in a very lightweight, water- and lighttight containment vessel to be submerged in the Wentworth pit. The $60 \mathrm{~m}$ depth provides an overburden of water to provide cosmic ray shielding at the same time as physically supporting the detector volume. This avoids the need to build a large structure or to excavate many kilotons of rock to contain the target water. The Cherenkov light from neutrino interaction remnants in the water will be collected by planes of $80 \mathrm{~mm}$ photomultiplier tubes (PMTs) positioned on the inner downstream side of the detector. It is therefore essential that the attenuation length of the Cherenkov light in the water be long enough to ensure very little reduction from production point to PMT. The pit once functioned as an open taconite mine and it is expected that the water will contain substantial suspended particulates. These can be removed by conventional filtering. The goal of this study was to understand whether, after filtering the remaining dissolved solids in the water would provide significant attenuation to the light. If this were to be case, further treatment of the water such as reverse osmosis or de-ionisation would be necessary, potentially leading to significant density differences between the inner and outer water, as the purified pit water will be used as the inner detector target mass. This could, in turn, lead to a significant increase in the cost, not only of the water treatment system, but also of the outer structure itself. The purification process and the method of the attenuation length measurement are reported here.

The attenuation length of the water from the Wentworth pit, made using a Secchi disk [2], is in the region of $2.5 \pm 0.5 \mathrm{~m}$. In order to achieve the 50 $100 \mathrm{~m}$ attenuation length required for a very large diameter detector [3], the proposal is to fill the detector with pit water that has been passed through a series of filters down to a Micron Rating of $0.2 \mu \mathrm{m}$. 


\section{Experimental Setup}

Pit water contained in a 50 (U.S.) gallon (189 litre) barrel is continuously passed through the cleaning system comprised of a UV sterilizer, a $1 \mu \mathrm{m}$ carbon filter, and a $0.2 \mu \mathrm{m}$ general purpose filter at a rate of 0.06 litres per minute (lpm) and circulated back into the barrel. The rate is set to simulate the rate of turnover in a large $30 \mathrm{~m}$ diameter CHIPS detector which will circulate $490 \mathrm{lpm}$ for each $2 \mathrm{~m}$ high detector slice. Generally, about 7 turnovers are required in order to ensure that $99 \%$ of the water has actually passed through the filters [4]. This would lead to a full water exchange approximately every two weeks.

A vertically mounted cylindrical tube of $3.2 \mathrm{~m}$ length and $50.8 \mathrm{~mm}$ (2 inch) inner diameter schedule 80 PVC pipe was sealed with a transparent perspex plate at the bottom through which a single $6 \mathrm{~mm}$ water pipe was inserted. This setup was inspired by the measuring equipment at University of California, Irvine [5] and is shown in Figure 1.

The fully automated measuring apparatus was controlled by a BeagleBone Black (BBB) [6] single-board computer running Linux. The column was filled with the water from the barrel via a servo operated valve controlled by one of the BBB Pulse Width Modulation pins to direct the water away from the filters and into the tube. The schematic of the water system is shown in Figure 2. The fill pump was allowed to run until the column contained a total water height of $3 \mathrm{~m}$, and was then stopped via a relay controlled by one of the BBB GPIO pins. A Thorlabs CPS405 collimated laser diode was mounted on a stage which allowed for adjustments in rotational and translational planes and was positioned to shine vertically upwards along the axis of the column. A lens with a focal length of $50 \mathrm{~mm}$ was inserted at the top of the column, $50 \mathrm{~mm}$ below a blue sensitive photo diode (1125-1009-ND 525NM) on axis at the top of the tube looking down at the laser.

The photodiode output was amplified using an operational amplifier circuit as shown in Figure 3. It was connected to the inputs of a trans-impedence amplifier, with feedback resistors of between 1 and $10 \mathrm{M} \Omega$ depending on the required gain so that the photodiode was operated in short circuit mode and not reverse biased. There was a voltage offset such that the output rested at around $50 \mathrm{mV}$ when the input current was zero. A clamping output buffer amplifier prevented the output from going above $1.8 \mathrm{~V}$ to avoid damage to the BBB input. The power source was a $9 \mathrm{~V}$ battery.

Once the column was full of water, the laser light was monitored in order 


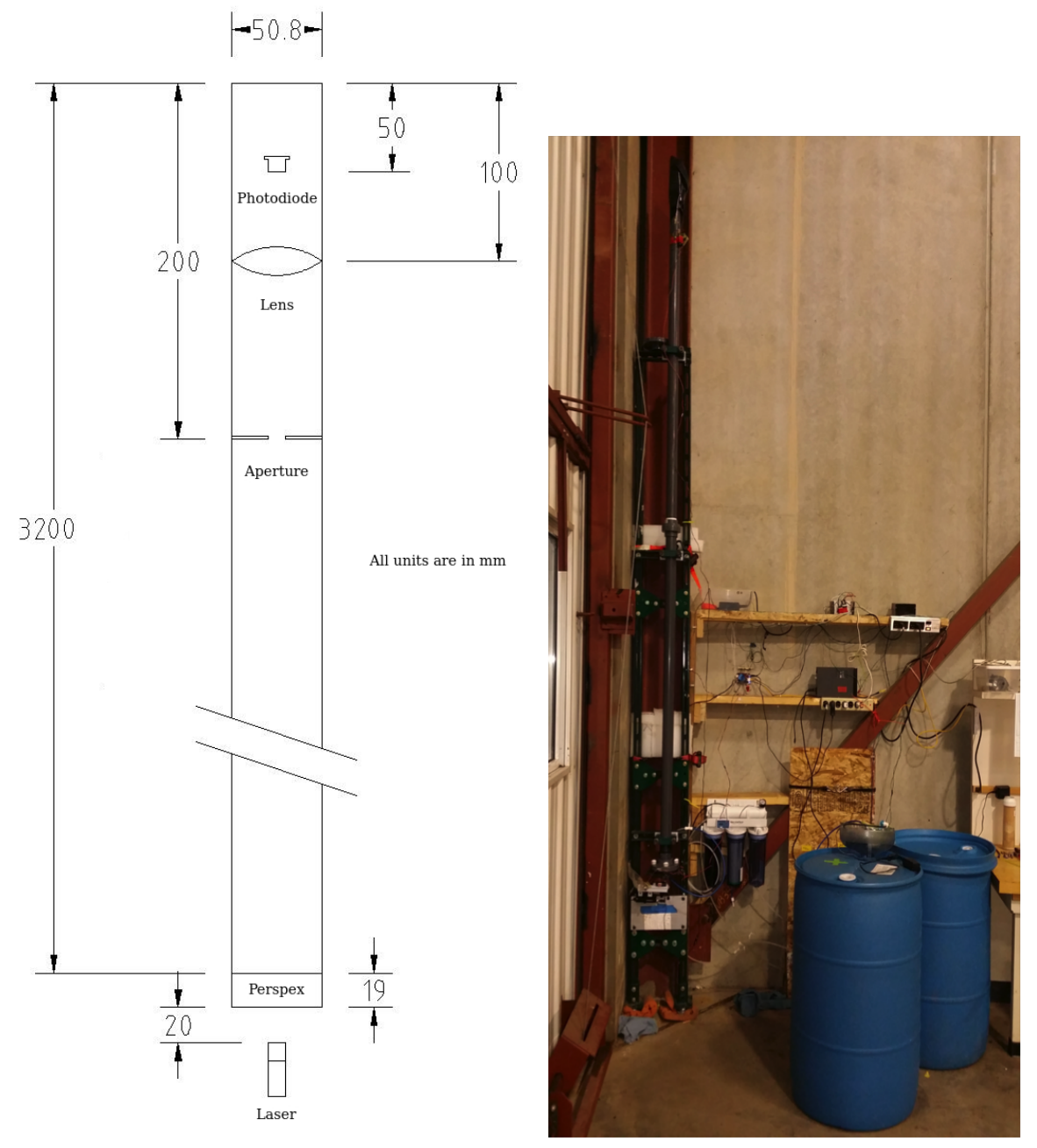

Figure 1: Left: schematic showing the key features of the water column (lengths in $\mathrm{mm}$ ) and right: photograph of the setup in the laboratory showing the column (left), the filters(centre) and the barrel (forefront right). 


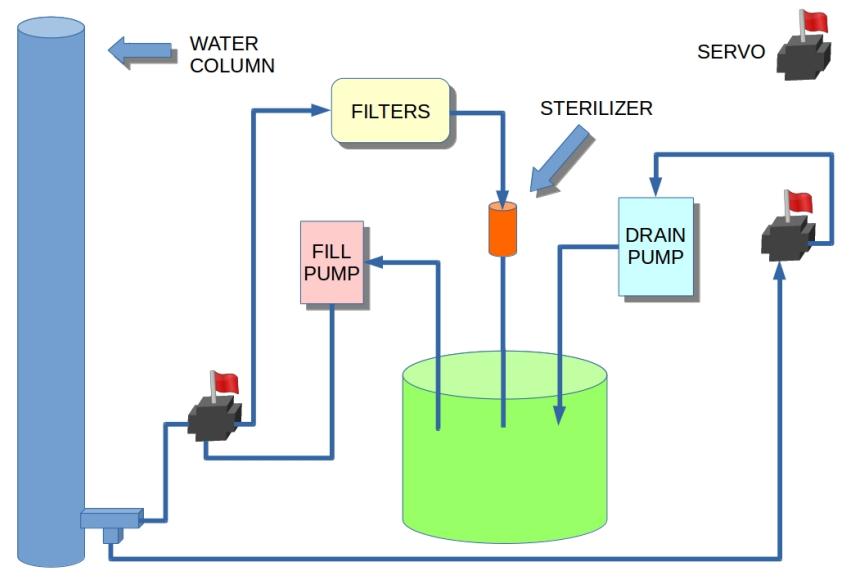

Figure 2: Sketch showing the layout of the pipework for the water circulation and the attenuation length measurement. The water continuously circulates until a measurement is performed at which point the servo direction is changed such that the column is filled before turning off the fill pump.

to estimate the time it would take for the bubbles to rise and the water to become stable after the turbulence caused by the fill pump. Figure 4 (left) shows the photodiode ADC as a function of time. The fill can be seen to occur where the ADC value falls to zero. A conservative period of 20 minutes was taken to allow for recovery. This is the time period from the fill point to the end of the $\mathrm{x}$ axis in the Figure. The light level can be seen to stabilize after this time. The next step in the process is to pump out a given amount of water. This also adds instantaneous vibration to the system, as shown in Figure 4 (right) where the two peaks correspond to the drain pump turning on and off and the recovery of the water clarity is seen in between. The BBB DAQ script was programmed to wait for 20 minutes after filling and at each measurement point.

The output from the photodiode circuit was digitized with one of the ADC channels on the BBB and data recorded in ASCII files for analysis. A total of 50 measurements was taken at each water height over a period of about 10 seconds. A quantity of water was then pumped out of the column and the procedure of pausing and taking data was repeated. The attenuation length was determined by measuring the intensity of light from the laser incident on the photodiode as a function of the height of water in the column and 

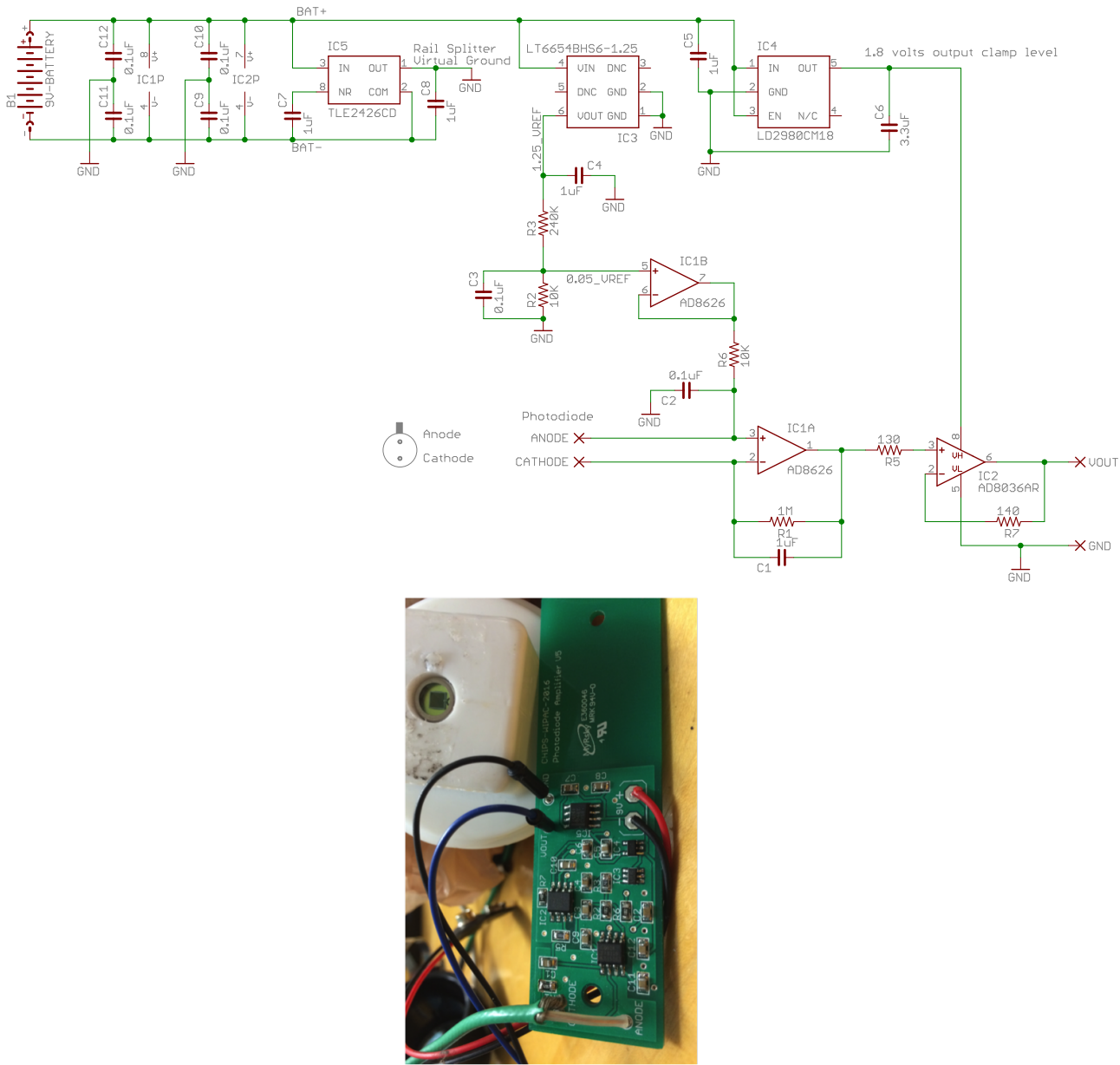

Figure 3: Amplifier circuit diagram (top) and fabricated board + photodiode (bottom) 

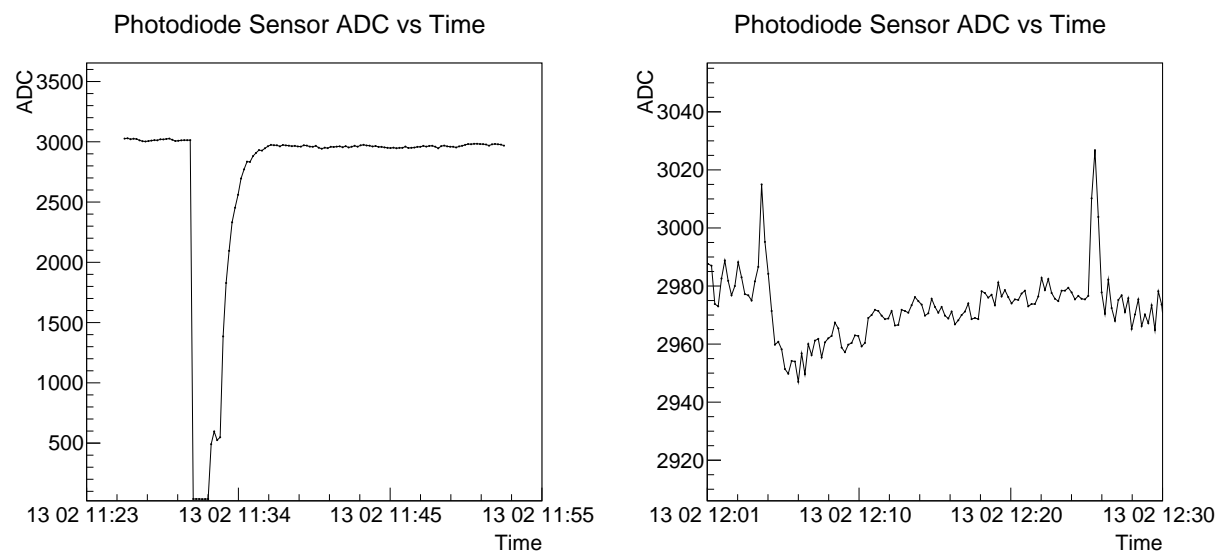

Figure 4: The left plot shows the photodiode ADC as a function of time. The fill is occurring where the ADC value has fallen to zero and the turbulence renders the water completely opaque. The right plot shows a time range after the fill is complete. When the drain pump turns on and off, the pipe shakes and this vibration causes turbulence in the water for a short time as evidenced by the peaks.

then performing an exponential fit to the graph. The error on the water height steps was less than $1 \mathrm{~cm}$; the fill time and emptying time were very repeatable. The number of steps could be varied by changing the amount of time the drain pump ran between data taking steps.

The water was circulated continuously through the filtration system in between taking regular attenuation length measurement runs. Improvements in the attenuation length were tracked as a function of time and therefore the effectiveness of the filtration and UV systems could be investigated.

\section{Data Analysis}

The intensity of the light at the top sensor is given by

$$
\mathrm{I}=\mathrm{I}_{0} \mathrm{e}^{-\mathrm{x} / \lambda}
$$

where $I_{0}$ is the light intensity with no water in the column, $\lambda$ is the attenuation length and $\mathrm{x}$ is the water height. The data are fit to extract the attenuation length.

The accuracy of the measurement is limited by the length of the column, the stability of the laser and the photodiode, the stability of the ambient temperature, the vibrational stability of the environment, the stability of the 
$\mathrm{BBB} \mathrm{ADC}$ and the amplifier circuit noise. There is therefore a limit to the maximum attenuation length measurement achievable.

The attenuation length in the $100 \mathrm{kt}$ detector is required to be at least $30 \mathrm{~m}$ at $405 \mathrm{~nm}$, as determined by reconstruction studies done on simulated neutrino interactions [3] but a longer attenuation length translates into more light at the PMTs and therefore better reconstruction of the interaction. Clearly, the longer the column, the more sensitive the measurement can be as it will increase the difference between the light levels when empty and full.

As an illustration, two examples are investigated. Taking the empty light level to be 3600 ADC counts in order to use most of the dynamic range of the 12 bit ADC, an attenuation length of $106 \mathrm{~m}$ would correspond to a drop in intensity to 3500 ADC counts (3\%) over a $3 \mathrm{~m}$ long length of water when the column was full. Similarly, an attenuation length of $52 \mathrm{~m}$ would correspond to a drop in intensity of $6 \%$ and the light level would measure 3400 ADC counts when full. Each attenuation length measurement involves $\mathrm{N} \approx 10$ separate measurements of the light level between full and empty which leads to an expectation of the uncertainty on the gradient to be the error on a single point divided by $\sqrt{N}$. With very long attenuation lengths $(>50 \mathrm{~m})$ a linear approximation works for the purposes of this illustration. Figure 5 shows the possible range of uncertainty produced by the pipe length limitation on the true attenuation length of $52 \mathrm{~m}$ and $106 \mathrm{~m}$ as a function of the single point error. The red lines show the lower limit and the blue lines show the upper limit. This provides a sanity check that a measurement of $100 \mathrm{~m}$ is safely far from the limitation of the $3 \mathrm{~m}$ column if the combined systematic error per point is kept at below about 30 ADC counts. Each contribution to the systematic error was measured as described below.

\subsection{Systematic Errors}

The contributions from the various sources of systematics were measured. As shown in Figure 6 the BBB ADC was measured to give a purely random jitter with a standard deviation of 1.1 ADC counts by measuring one of the BBB ADC channels connected to a simple voltage divider circuit. The laser specification gives a stability of the laser output power of $1 \%$ over a 1 minute period and $2 \%$ over an 8 hour period. The attenuation length measurement run time is between one and two hours, so a systematic of $1.5 \%$ for the overall stability during this time period might be expected.

The photodiode and laser stability are measured together by monitoring the ADC output as a function of time for a full water column. In addition 


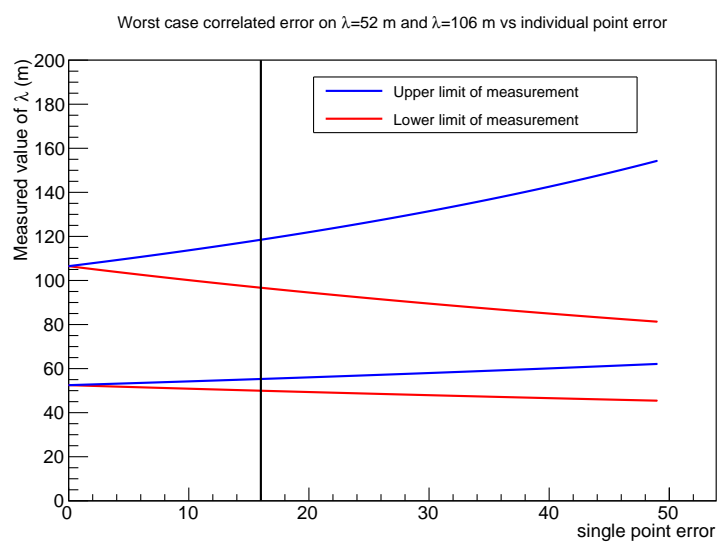

Figure 5: An illustration of the effect of the single point uncertainty on the measurement of the attenuation length, assuming a true attenuation length of $52 \mathrm{~m}$ and $106 \mathrm{~m}$. In the case of a 16 ADC count error on each point (as measured in Section 3.3), shown by the vertical line, the attenuation length could be mis-measured by up to $2.5 \mathrm{~m}$ and up to $12 \mathrm{~m}$ respectively

ambient vibrations which affect the full water column are folded in to this measurement. Figure 7 shows the dependence of the photodiode output with temperature over a period of a few hours. The temperature was found to have the largest impact on the system stability of all the systematics.

\subsection{Cleaning Rate Measurement}

Once the water was being passed through the filtration system, samples were taken regularly from the barrel to observe how the attenuation length increased with time. The temperature was continuously monitored throughout this procedure and used to estimate the errors on the data points for each attenuation length measurement. Initial measurements of the unfiltered pit water can be seen in Figure 8 (top left), where the attenuation length was measured to be $1.6 \pm 0.05 \mathrm{~m}$. The result is in reasonable agreement with the measurement with the Secchi disk for white light. Other results can be seen after 12 days (top right) and after 25 days(bottom).

Figure 9 shows the outcome of the measurements over a four month period. Larger errors are associated with measurements during the colder months when the laboratory heater cycles produced large temperature related variations in the ADC output. The temperature was the dominant systematic delivering an uncertainty over the course of each attenuation length 


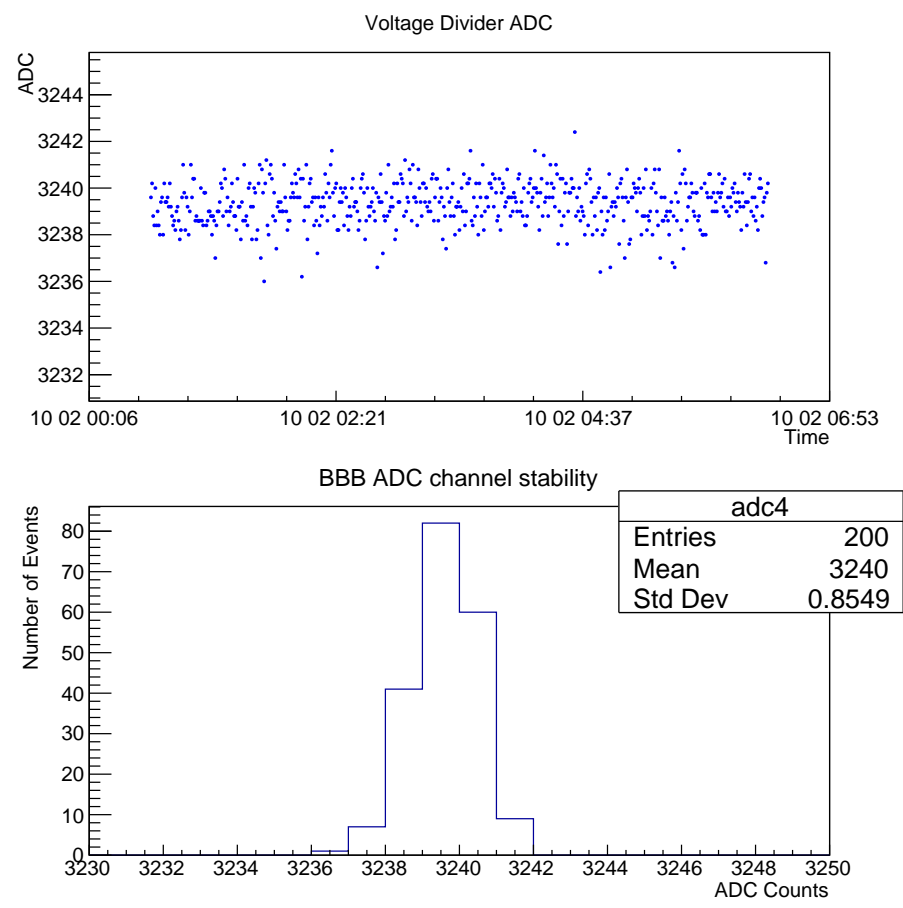

Figure 6: Top: BBB ADC channel reading from a voltage divider as a function of time. Bottom: the projection of the time evolution showing a standard deviation of $1.1 \mathrm{ADC}$ counts. 


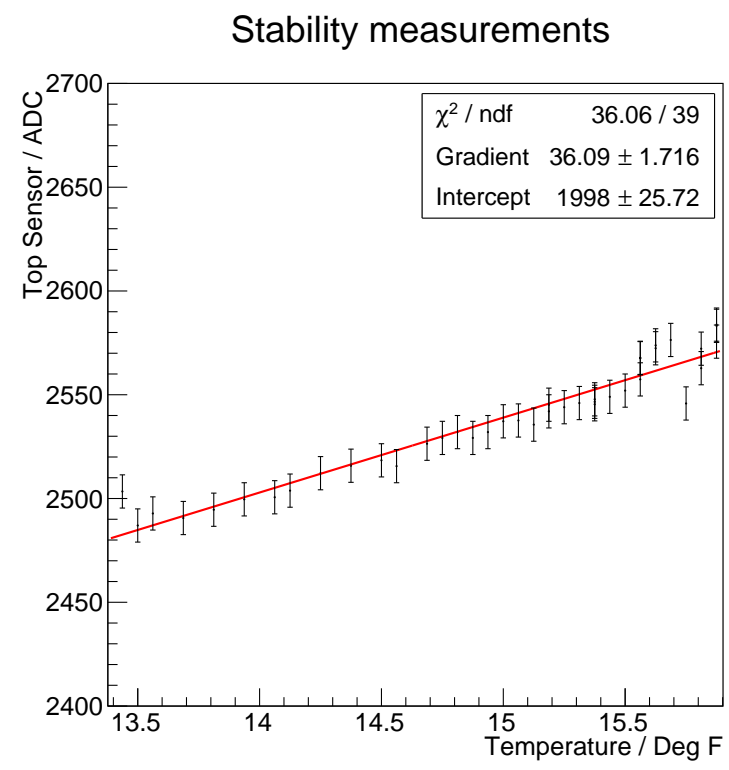

Figure 7: Top: Photodiode ADC as a function of temperature for a full water column taken over a period of a few hours. A linear fit shows a change of about 38 ADC counts per ${ }^{\circ} \mathrm{C}$. Continuous temperature monitoring was used to estimate the size of the errors on the data points for a given attenuation length measurement run.

measurement of sometimes up to 38 ADC counts per point for a change between single point measurements of $1^{\circ} \mathrm{C}$. However, it can be seen that the filtration system is effective with a clearly increasing trend in attenuation length up to values of $50-60 \mathrm{~m}$, and referring back to Figure 5 within the accuracy of the measurement system. This suggests a relatively simple filtration system will be adequate for a detector size of $30 \mathrm{~m}$ diameter. It can also be seen that a circulation time of about 3-4 months should provide an attenuation length of $50 \mathrm{~m}$.

\subsection{System Improvements}

Once the time constant of the water cleaning had been determined, steps were taken to make the setup more stable to improve the accuracy of the measurements at longer attenuation length and to ascertain whether further cleaning would be possible. This was done by insulating the electronics and laser from temperature changes, increasing the water recirculation speed to clean the water much faster, and working in April when the temperature was warm enough to avoid the heater cycles. It is possible that an additional 

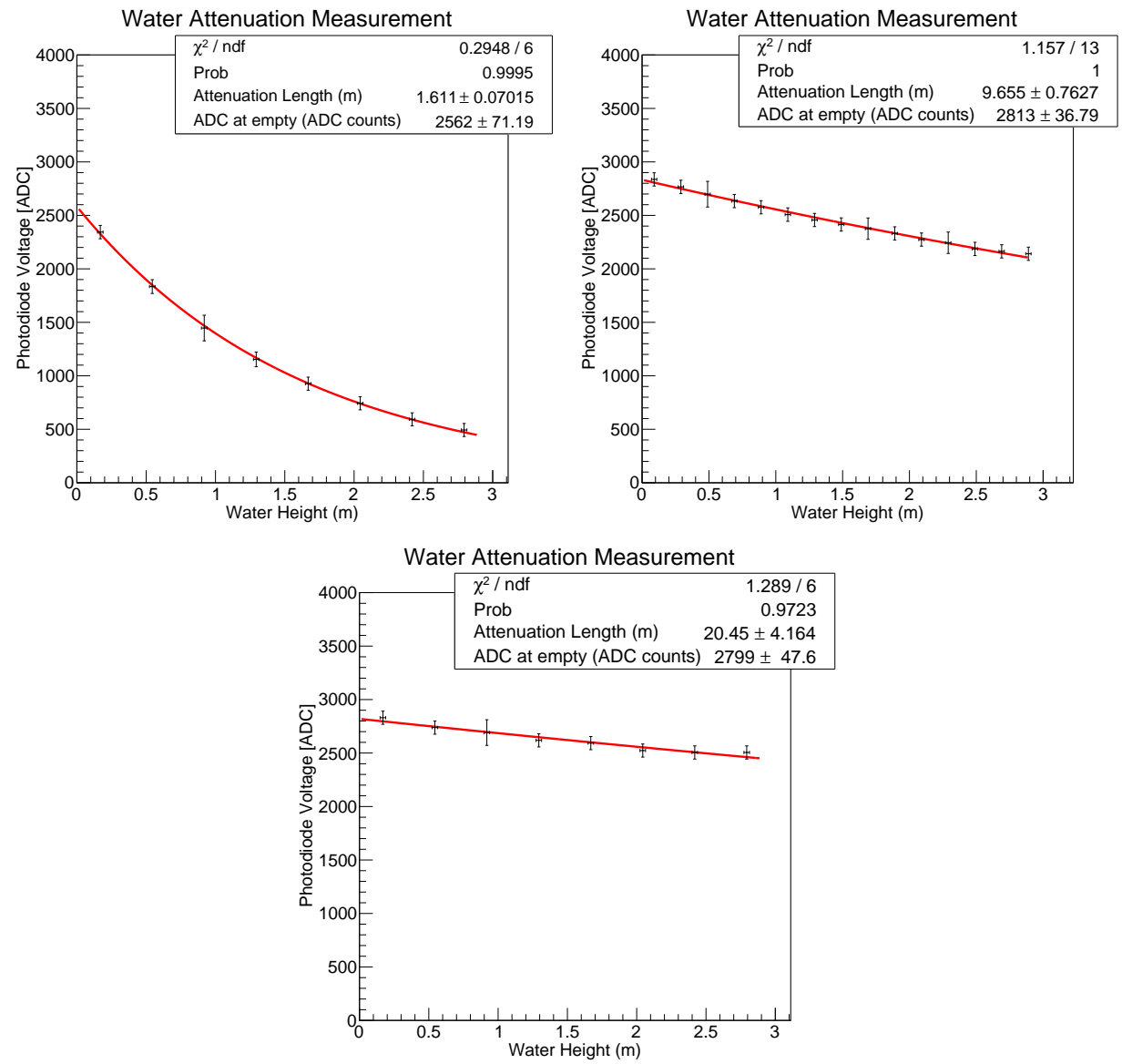

Figure 8: Plot showing water attenuation measurement for raw pit water (top left); after filtering for 12 days (top right) and after filtering for 25 days (bottom) 
Water Attenuation Time Evolution

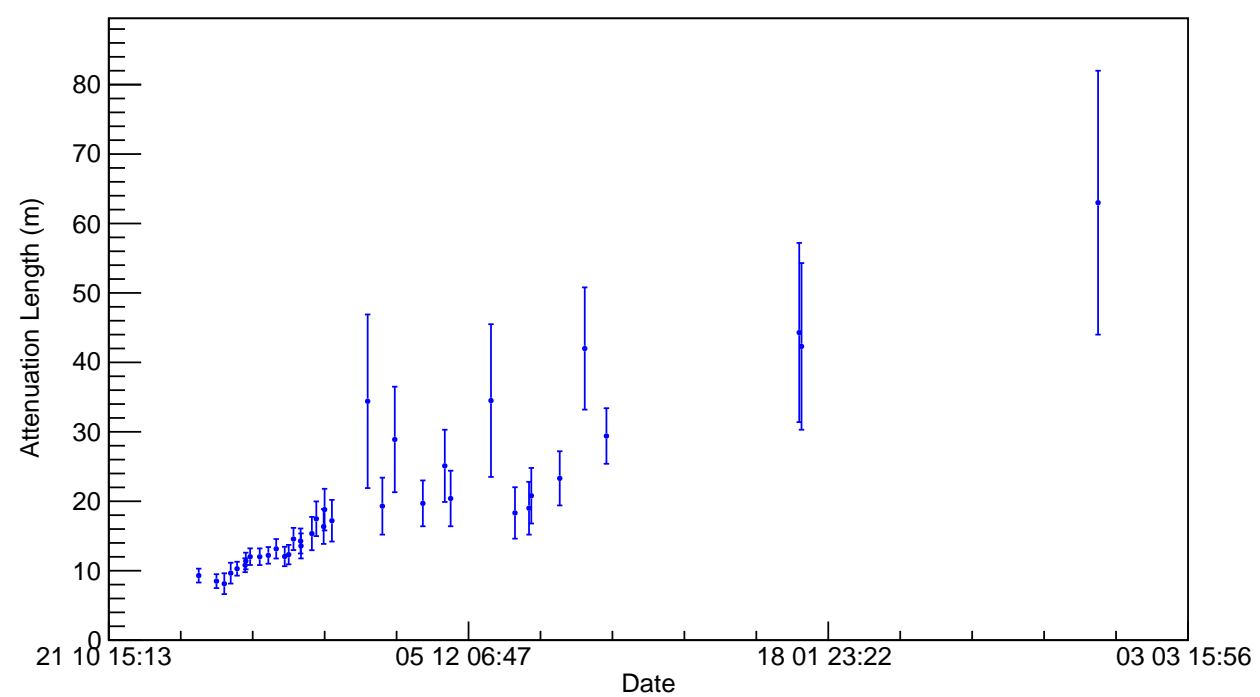

Figure 9: Plot showing how attenuation length improved with time while the water was continuously circulated through the filtration system.

speed up in the water clarity with recycle rate was due to there being no chance for any bacteria to survive such short circulation times, although this is conjecture.

Figure 10 (top) shows the photodiode ADC for a full water column in the improved set up as a function of time. The lower histogram shows the projection with a standard deviation of 15.7 ADC counts. This measurement incorporates all of the systematic uncertainties and results in an overall uncertainty of $0.5 \%$. While this is smaller than the specification for the laser stability, the measured systematic error of 16 ADC counts is nevertheless applied to each data point in the subsequent attenuation length fit procedure. This is well below the target of 30 ADC counts described above for attenuation lengths above $50 \mathrm{~m}$.

\subsection{Final Results}

Figure 11 shows three attenuation length measurements taken at intervals of $0,1.5$ and 12 hours of water circulation with attenuation lengths of $\lambda=1.68 \pm 0.02 \mathrm{~m}, 22.4 \pm 1.2 \mathrm{~m}$ and $139 \pm 42 \mathrm{~m}$ respectively. This increase in speed of water cleaning is just due to the increased circulation rate of the water through the filters and sterilizer. 

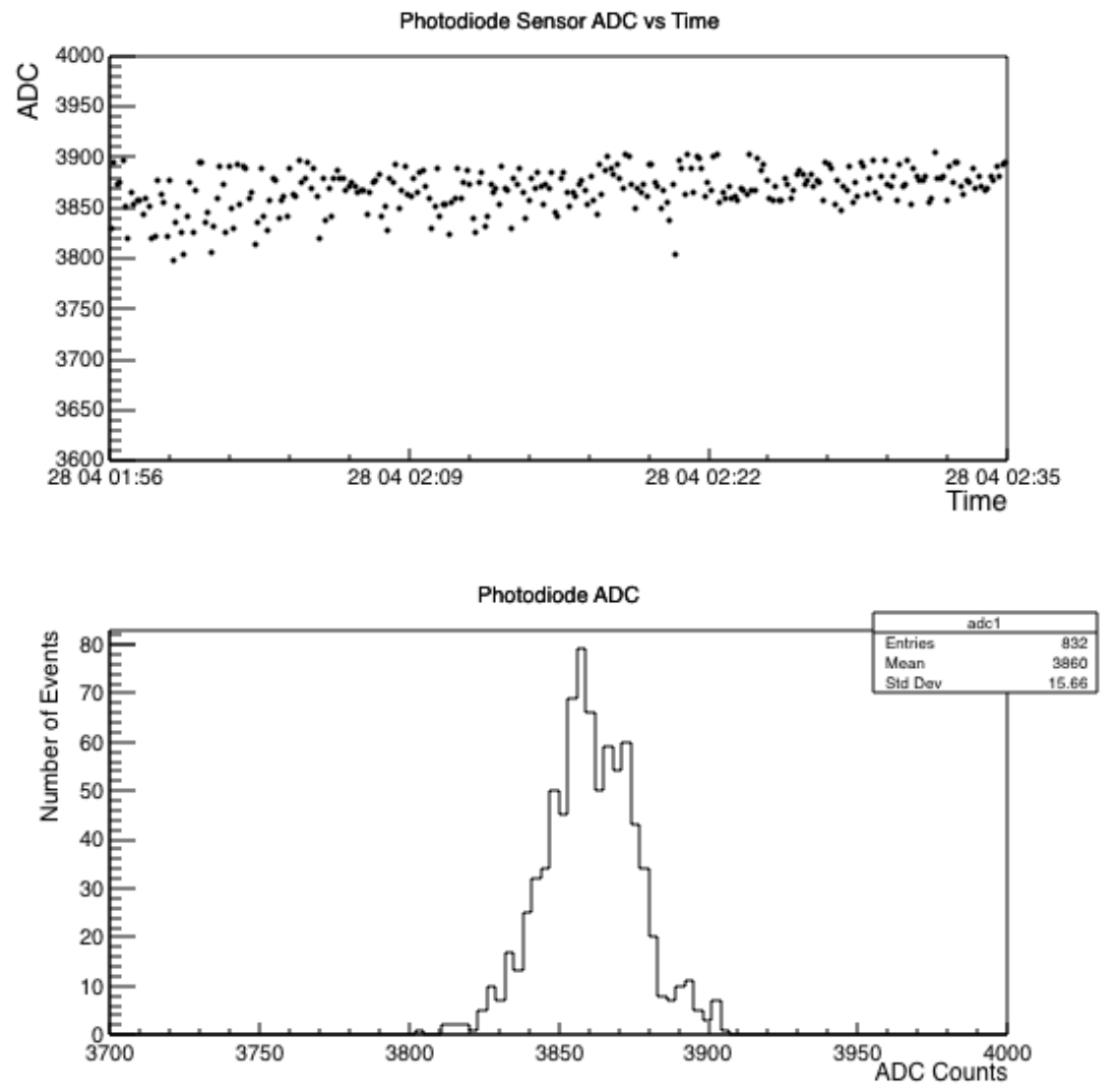

Figure 10: Top: ADC + photodiode stability after system improvements. Bottom: the projection of the ADC values showing an RMS of 15.6 ADC counts.

\section{Simulation}

A dedicated simulation program was developed to cross check and disentangle any contributions to the attenuation length measurement from the optical setup. The simulated photon's trajectory was traced according to the rules of geometrical optics and could be absorbed in the water with a probability depending on the water absorption length. A photon was considered as detected if it reached the photodetector. The simulation made use of the ROOT [7] software framework but all the code describing the photon transportation and interaction processes was developed independently. All the optical elements, including the focussing lens in front of the photodiode, and 
Water Height vs ADC

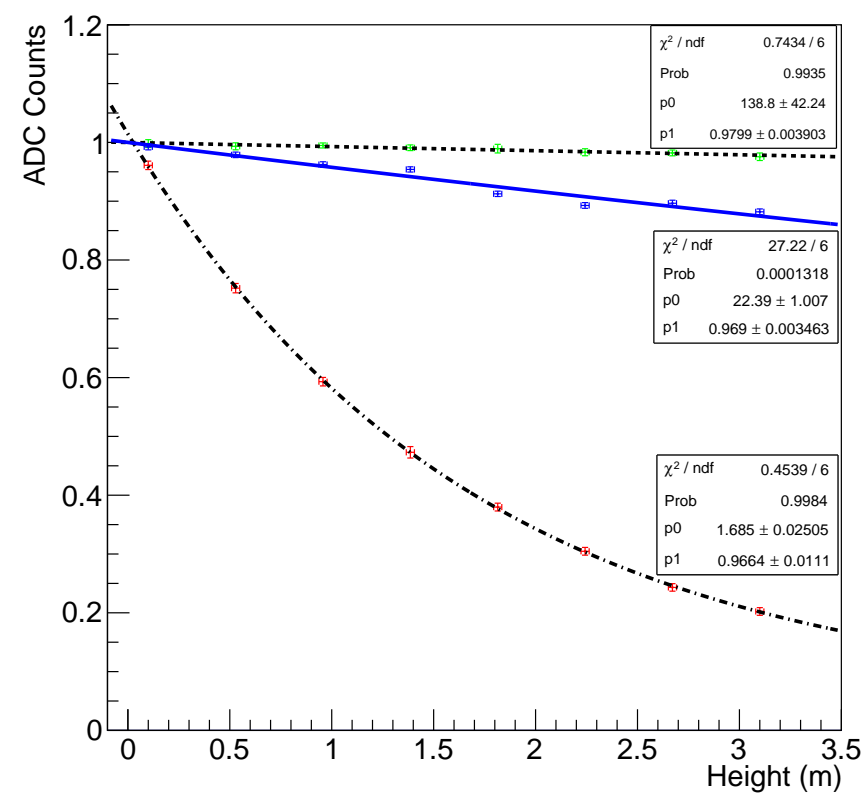

Figure 11: Plot showing attenuation length measurements for three different levels of water clarity, at the start (dot-dash), after 1.5 hours(solid) and after 12 hours(dashed). The y axes have been normalised for comparison to take out longer term drift as described.

the material boundaries present in the experimental setup were considered in the photon ray-tracing; the laser beam width and divergence were modelled according to the Thorlabs data sheet [8]. The geometrical parameters for the laser (direction, starting position) and the photodiode (position, size) could all be individually varied. The water level and its attenuation length could also be varied. The beam divergence is very small, and measurements made with the above setup were unable to detect any light more than $5 \mathrm{~mm}$ away from the laser center. Therefore it was deemed unnecessary to simulate any scattered light from the inside of the tube as our measurements showed it would not be possible to detect such a low light level.

Given a random starting position and direction each photon was propagated through every material boundary, where Snell's Law was applied, up to the photodetector plane. The focussing lens was modelled by applying refraction at the spherical surfaces.

The simulated data points and the fit to them showed there were no 

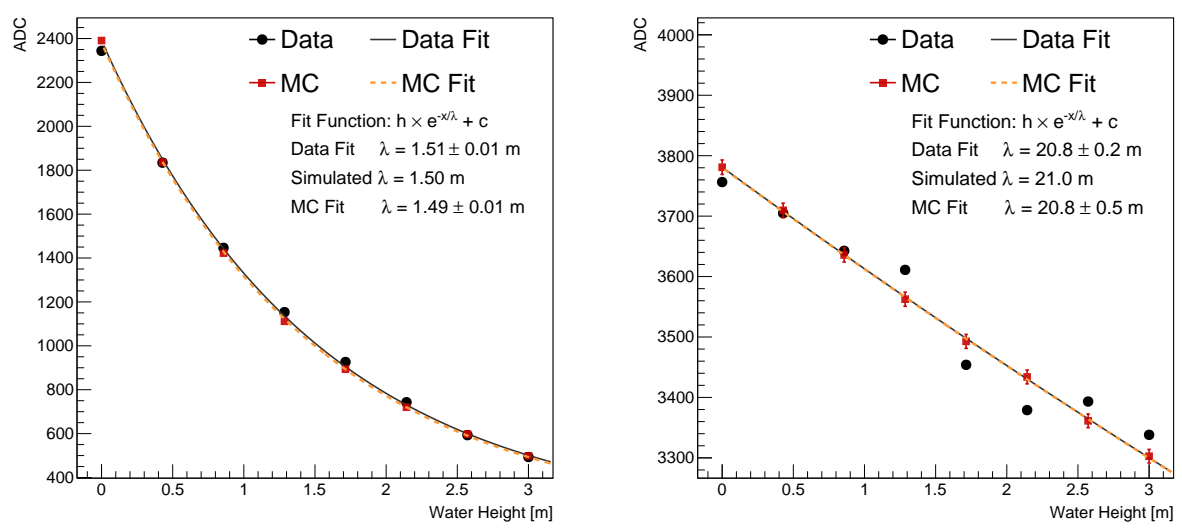

Figure 12: Left: light intensity measured by the photodiode expressed in ADC counts, as a function of the water level for experimental data (dots) and simulation (squares). The attenuation length $(\lambda)$ extracted from a fit (solid line) to the data points is $\lambda=$ $1.51 \pm 0.01 \mathrm{~m}$. Also shown is the simulation with an input value of $\lambda=1.5 \mathrm{~m}$ whose corresponding fit (dashed line) gives $\lambda=1.49 \pm 0.01 \mathrm{~m}$. Statistical errors only are used. Right: a longer attenuation length of $\lambda=20.8 \pm 0.2 \mathrm{~m}$ for the data and the input to the simulation of $\lambda=21 \mathrm{~m}$ shows the corresponding fit of $\lambda=20.8 \pm 0.5 \mathrm{~m}$.

unexpected systematics associated with the optical geometry: for example the refractive index changes from air to perspex, to water, to air, to glass and to lens did not produce any effect which could be mistaken for a change in attenuation length. The simulation results are summarised in Figure 12 where it is shown that the relative attenuation length fits are both in good agreement with the results from the experimental data and demonstrate no unaccounted for systematic effects for both short and medium attenuation length.

\section{Conclusions}

The attenuation length of the water from the Wentworth Pit 2W, site of the CHIPS experiment, has been measured during filtration. The UV sterilization and simple filtering method was found to be capable of delivering in the region of $100 \mathrm{~m}$ attention length at a wavelength of $405 \mathrm{~nm}$. This is completely adequate for a $30 \mathrm{~m}$ diameter CHIPS detector as shown by detailed simulation and reconstruction studies. Without the need for the exclusion of dissolved solids, the operational complexity and cost of the water treatment 
[1] Cherenkov detectors In mine PitS (CHIPS) Letter of Intent to FNAL, arXiv 1307.5918 (2013)

[2] https://en.wikipedia.org/wiki/Secchi_disk

[3] Neutrino 2016 proceedings, J.Phys.: Conf. Ser. to be published.

[4] Clay's Handbook of Environmental Health, p272

[5] Micheal Smy, priviate communication 2015.

[6] https://beagleboard.org/black

[7] R. Brun, F. Rademakers, "ROOT - An Object Oriented Data Analysis Framework", Proceedings AIHENP'96 Workshop, Lausanne, Sep. 1996, Nucl. Inst.\& Meth. in Phys. Res. A 389 (1997) 81-86. See also http://root.cern.ch/.

[8] https://www.thorlabs.com/thorproduct.cfm?partnumber=CPS405 